\title{
Evaluation of a patient self-stratification methodology to identify those in need of shielding during COVID-19
}

\author{
Authors: Elizabeth Reilly, ${ }^{A}$ Sarah Skeoch, ${ }^{B}$ Sarah Hardcastle, ${ }^{\mathrm{B}}$ John D Pauling, ${ }^{\mathrm{C}}$ Megan Rowe, ${ }^{\mathrm{D}}$ Tehseen Ahmed, ${ }^{\mathrm{B}}$ \\ Andrew Allard, ${ }^{B}$ Bashaar Boyce, ${ }^{B}$ Ellie Korendowych, ${ }^{B}$ Chloe Lapraik, ${ }^{B}$ William Tillett ${ }^{C}$ and Raj Sengupta ${ }^{B}$
}

\begin{abstract}
The logistical challenges of rapidly and accurately identifying those patients who needed to shield during the COVID-19 pandemic were unprecedented. We report our experiences of meeting this challenge for $>\mathbf{9 , 0 0 0}$ patients with rheumatic and musculoskeletal disease at our centre, incorporating an element of guided patient self-stratification. Our results indicate that patients are able to stratify their own risk accurately using the BSR COVID-19 risk stratification guidance.
\end{abstract}

KEYWORDS: COVID-19, rheumatic and musculoskeletal disease, service evaluation, British Society for Rheumatology

DOI: $10.7861 /$ clinmed.2020-0469

\section{Introduction}

Coronavirus 2019 (COVID-19) has presented a unique constellation of clinical, logistical and ethical challenges for rheumatology. There have been concerns that patients with rheumatic and musculoskeletal disease (RMD) receiving conventional synthetic (cs-), biological (b-) or targeted synthetic (ts-) disease modifying anti-rheumatic drugs (DMARDs) may have an increased risk of COVID-19, based upon previous experience in this population with infectious diseases. ${ }^{1,2}$ Furthermore, patients with multisystem diseases such as connective tissue diseases (CTDs) and vasculitis may be at particular risk in view of their potential co-existent respiratory or renal compromise. ${ }^{3-5}$ The need to urgently identify those at highest risk and provide them with guidance was supported by the publication of guidance and risk assessment tools by NHS England and the British Society for Rheumatology (BSR). ${ }^{6,7}$ These documents provided a framework to approach risk assessment and stratification, with those designated 'high risk'

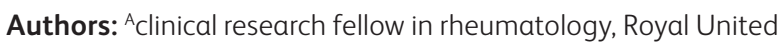
Hospitals Bath NHS Trust, Bath, UK and University of Bath, Bath, UK; ${ }^{B}$ consultant rheumatologist, Royal United Hospitals Bath NHS Trust, Bath, UK; ' Consultant rheumatologist, Royal United Hospitals Bath NHS Trust, Bath, UK and senior lecturer, University of Bath, Bath, UK; Drheumatology pharmacist, Royal United Hospitals Bath NHS Trust, Bath, UK given advice on shielding. While early signals from Wuhan, China, and Italy had so far appeared to demonstrate reassuringly low numbers of cases of COVID-19 in those with immunodeficiency or on immunosuppressants, BSR produced clear guidance that these factors should be considered relevant in assessing patients' risks of COVID-19 until further, unequivocal evidence demonstrated otherwise, a view held by many clinicians, and hence their stratification process was widely adopted.

\section{Methods}

The logistical challenges of undertaking this risk stratification rapidly in a large population were unprecedented. ${ }^{8}$ We report our experiences of meeting this challenge for $>9,000$ RMD patients managed at our centre, based upon the BSR guidance for identifying those at high risk of COVID-19 and in need of shielding. Our principal aim was to deliver accurate and timely risk-stratification for COVID-19 precautionary measures for a large cohort of patients.

In order to complete this task quickly and efficiently, we took a stepwise approach:

> Undertake individualised assessment of those on bDMARDs and advise them of their personalised risk and related guidance.

> Contact patients with CTD and vasculitis, advising them to shield, accepting some low-moderate risk patients would be included.

> Contact patients with CTD-associated pulmonary arterial hypertension (PAH) and/or interstitial lung disease (ILD) with advice to shield.

$>$ Devise a risk stratification tool to be sent out to all remaining RMD patients enabling them to score their own risk level accurately.

We initially prioritised assessment of those on biologics, identified through our biologics database, and their risk was scored by departmental clinicians. CTD and vasculitis cases were identified through electronic patient record (EPR) text mining and clinic lists, and shielding letters were sent accordingly. ${ }^{9}$ A total of 1,622 patients on bDMARDS and tsDMARDS and 474 patients with CTD/vasculitis were sent letters with their individualised risk stratification by 7 April 2020.

In order to reach other potentially at-risk patients from our cohort with RMD, a further 7,517 patients with RMD registered 
Table 1. Results of patients' self-scoring using both the paper and web based COVID19 scoring methods

$\begin{array}{llll}\text { Scoring method } & \begin{array}{l}\text { High risk } \\ (\text { Shielding) } \mathbf{n}(\%)\end{array} & \begin{array}{l}\text { Moderate risk (Strict social } \\ \text { distancing) } \mathbf{n}(\%)\end{array} & \begin{array}{l}\text { Low risk (Standard social } \\ \text { distancing) } \mathbf{n}(\%)\end{array} \\ \text { Online risk matrix } & 99(11 \%) & 208(23 \%) & 603(66 \%) \\ \text { Paper risk matrix } & 165(18 \%) & 281(31 \%) & 464(51 \%)\end{array}$

under our care within the last 2 years were identified through EPR text mining of clinical correspondence. They were sent comprehensive information guiding them through a process of self-stratification based on BSR guidance. There were two parts to this: a paper scoring method (supplementary material S1) with instructions for the patient to work through and a link to an internet platform (supplementary material S2). Our online platform guided patients through the same risk stratification matrix to identify their risk group and also prompted patients to enter the score they had calculated using the paper risk matrix. The latter method enabled us to capture patients' self-scoring and add them to the central list of shielding patients. To validate the reliability of the patient self-scoring method, 100 consecutive patients were contacted in telephone clinics and asked how they scored themselves. They were then re-scored by a rheumatologist.

\section{Results}

Of 100 patients contacted by telephone, 97 had received the letter and stratified their risk. Of these, 89 had estimated their risk correctly and 31 had not assessed themselves using the web portal. The reasons given for not using the web portal were that they felt sufficiently informed already or that it was too complex. Only $5 \%$ of this sample were required to shield, offering a basis on which to estimate the numbers needing to shield in our wider RMD population.

Of the 7,517 RMD patients who were sent guidance on self-scoring, 910 (13\%) logged onto the web platform to complete the online process over the first 4 weeks. Table 1 shows how patients scored themselves using each method; $72 \%$ scored themselves consistently by both methods.

\section{Discussion}

We report a systematic approach to contacting our RMD patient cohort with personalised guidance to help them protect themselves during the COVID-19 pandemic, based upon the process produced by BSR. Our stepwise process enabled us to issue prompt guidance for those at the highest risk and adopt a more nuanced assessment for those with other risk factors. Our followup telemedicine reviews indicated that most patients felt they had been supplied with sufficient information via letter to safely manage their risk. Those using the web portal represented a small proportion of our patient cohort, with inherent selection bias. Limited patient engagement in this sort of initiative has also been reported by others. ${ }^{10}$ In comparison, we report a higher proportion of engagement in those in the low risk group, and fewer in the medium or high-risk categories, likely owing to our supplementary approach for those on bDMARDs and with CTD. Nonetheless, our analysis suggests patients could apply the risk stratification algorithms effectively. While it is acknowledged that by using this method, some patients would have been over-scored, it was felt that on balance it was most important to minimise the risks of under-advising those in the highest risk categories. However, we appreciate there were some unintended implications, such as the potential negative impact on mental health, ${ }^{11}$ for those advised to shield without cause.

Further work on identifying barriers to engagement in webbased patient reporting is required but could provide an excellent way of assessing disease and risks remotely at a time where face-to-face contact needs to be minimised. Barriers encountered in trying to carry out this process in a timely and accurate way included staff re-deployment to acute medical duties, meaning manual scoring of all RMD patients under our care was not feasible, and limited EPR coding, particularly of patients on combination DMARDs or high-dose corticosteroids. This suggests the need for new, innovative methods (such as mobile-phone based systems), consensus agreement on the minimum data capture for clinical databases and the development of fully integrated technology to facilitate contacting patients quickly should the need arise due to future pandemics.

\section{Conclusion}

We report the application of a multi-layered approach comprising individual case note review, rule-based methods for certain diseases (eg PAH and ILD), and postal/web-based patient selfstratification to promptly risk stratify $>9,000$ RMD patients from a single centre. Our results indicate that patients are able to stratify their own risk accurately using the BSR COVID-19 risk stratification guidance, enabling them to take precautionary measures to modify their risk of contracting COVID-19. While we cannot be sure that our efforts have led to complete coverage of those who need to shield, an analysis of data from the NHS spine has reassured us that, at the time of writing, no patient on bDMARDS or tsDMARDS from our cohort has died of COVID-19.

\section{Supplementary material}

Additional supplementary material may be found in the online version of this article at www.rcpjournals.org/clinmedicine: S1 - Paper patient COVID-19 risk stratification letter S2 - Online patient COVID-19 risk stratification form

\section{Acknowledgements}

Deborah Bond, Janet Ball and Nicola Fulstow, clinical nurse specialists at the Royal National Hospital for Rheumatic Diseases, for their help with data collection.

\section{Conflicts of interest}

Elizabeth Reilly reports research grants from Actelion and Celgene, outside of the submitted work. John Pauling reports research grants, personal fees and non-financial support from Actelion Pharmaceuticals. Dr Pauling also reports personal fees from 
Boehringer Ingelheim; and personal fees from Sojournix Pharma, outside the submitted work. Raj Sengupta reports research grants, consultancy and speaker fees from Abbvie, Biogen, Celgene, Lilly, Novartis and UCB, outside the submitted work. Sarah Skeoch reports grants from Innovative Medicines Initiatives 2 Joint Undertaking, and personal fees from Pfizer, outside the submitted work.

\section{References}

1 Furst DE. The risk of infections with biologic therapies for rheumatoid arthritis. Semin Arthritis Rheum 2010;39:327-46.

2 Kourbeti IS, Ziakas PD, Mylonakis E. Biologic therapies in rheumatoid arthritis and the risk of opportunistic infections: a meta-analysis. Clin Infect Dis 2014;58:1649-57.

3 Kahl LE. Herpes zoster infections in systemic lupus erythematosus: risk factors and outcome. J Rheumatol 1994;21:84-6.

4 Tektonidou MG, Wang Z, Dasgupta A, Ward MM. Burden of serious infections in adults with systemic lupus erythematosus: a national population-based study, 1996-2011. Arthritis Care Res (Hoboken) 2015;67:1078-85.

5 Feldman CH, Hiraki LT, Winkelmayer WC et al. Serious infections among adult Medicaid beneficiaries with systemic lupus erythematosus and lupus nephritis. Arthritis Rheumatol 2015;67:1577-85.

6 British Society for Rheumatology. COVID-19 - Identifying patients for shielding in England. BSR, 2020. www.rheumatology.org.uk/ Portals/O/Documents/Rheumatology_advice_coronavirus_immuno suppressed_patients_220320.pdf?ver=2020-03-24-171132-407.
7 Price E, MacPhie E, Kay L et al. Identifying rheumatic disease patients at high risk and requiring shielding during the COVID-19 pandemic. Clin Med 2020;20:256-61.

8 Mikuls TR, Johnson SR, Fraenkel L et al. American College of Rheumatology guidance for the management of adult patients with rheumatic disease during the COVID-19 pandemic. Arthritis Rheumatol 2020;72:1241-51.

9 NHS England. Clinical guide for the management of rheumatology patients during the coronavirus pandemic. (Version 1.) NHSE, 2020. www.england.nhs.uk/coronavirus/wp-content/uploads/ sites/52/2020/03/clinical-guide-rheumatology-patients-v1 19-march-2020.pdf.

10 Bateman J, Mulherin D, Hirsch G, Venkatachalam S, Sheeran T. Rapid distribution of information by SMS-embedded video link to patients during a pandemic. Lancet Rheumatol 2020;2:e315-6.

11 Michaud K, Wipfler K, Shaw Y et al. Experiences of patients with rheumatic diseases in the United States during early days of the COVID-19 pandemic. ACR Open Rheumatol 2020;2:335-43.

Address for correspondence: Dr Elizabeth Reilly, Royal National Hospital for Rheumatic Diseases, Royal United Hospitals Bath NHS Trust, Combe Park, Bath BA1 3NG, UK.

Email: elizabethreilly2@nhs.net 